DRAFT VERSION JUNE 4, 2018

Preprint typeset using LTEX style emulateapj v. 5/2/11

\title{
STOCHASTIC HEATING, DIFFERENTIAL FLOW, AND THE ALPHA-TO-PROTON TEMPERATURE RATIO IN THE SOLAR WIND
}

\author{
B. D. G. Chandran ${ }^{1,4}$, D. Verscharen ${ }^{1}$, E. Quataert ${ }^{2}$, J. C. KASPer ${ }^{3}$, P. A. Isenberg ${ }^{1}$, \& S. Bourouaine $^{1}$ \\ Draft version June 4, 2018
}

\begin{abstract}
We extend previous theories of stochastic ion heating to account for the motion of ions along the magnetic field $\boldsymbol{B}$. We derive an analytic expression for the temperature ratio $T_{\perp \mathrm{i}} / T_{\perp \mathrm{p}}$ in the solar wind assuming that stochastic heating is the dominant ion heating mechanism, where $T_{\perp \mathrm{i}}$ is the perpendicular temperature of species $\mathrm{i}$ and $T_{\perp \mathrm{p}}$ is the perpendicular proton temperature. This expression describes how $T_{\perp \mathrm{i}} / T_{\perp \mathrm{p}}$ depends upon $U_{\mathrm{i}}$ and $\beta_{\| \mathrm{p}}$, where $U_{\mathrm{i}}$ is the average velocity along $\boldsymbol{B}$ of species $\mathrm{i}$ in the proton frame and $\beta_{\| \mathrm{p}}$ is the ratio of the parallel proton pressure to the magnetic pressure, which we take to be $\lesssim 1$. We compare our model with previously published measurements of alpha particles and protons from the Wind spacecraft. We find that stochastic heating offers a promising explanation for the dependence of $T_{\perp \alpha} / T_{\perp p}$ on $U_{\alpha}$ and $\beta_{\| \mathrm{p}}$ when the fractional cross helicity and Alfvén ratio at the proton-gyroradius scale have values that are broadly consistent with solar-wind measurements. We also predict how the temperatures of other ion species depend on their drift speeds.
\end{abstract}

Subject headings: solar wind — Sun: corona — turbulence — waves

\section{INTRODUCTION}

As solar-wind plasma flows away from the Sun, it moves into regions of progressively weaker magnetic field. If the magnetic moments $\mu$ of solar-wind ions were conserved, then the perpendicular temperature $T_{\perp}$ of each ion species would be a strongly decreasing function of heliocentric distance $r$. However, in situ spacecraft measurements show that $T_{\perp}$ decreases much more slowly with $r$ than $\mu$ conservation would imply, indicating that ions undergo some form of perpendicular heating (Marsch et al. 1982a. b; Kohl et al. 1998).

One model for explaining this heating invokes resonant cyclotron heating by Alfvén/ion-cyclotron (A/IC) waves (Hollweg \& Isenberg 2002). Cyclotron heating violates $\mu$ conservation and offers a possible explanation for the observed preferential heating of minor ions (Isenberg \& Vasquez 2007) as well as the shape of the core of the proton velocity distribution in fast-solar-wind streams (Galinsky \& Shevchenko 2000; Isenberg et al. 2001; Marsch et al. 2004; Isenberg \& Vasquez 2011). The primary difficulty faced by cyclotron-heating models is that it is not clear that high-frequency A/IC waves can be produced in sufficient quantities to explain the levels of proton heating that are observed. Early studies postulated that a turbulent cascade efficiently transfers the energy of non-compressive, magnetohydrodynamic (MHD) fluctuations from large scales to small scales and from low frequencies to high frequencies (Isenberg \& Hollweg 1983; Isenberg 1984; Tu et al. 1984; Hollweg 1986; Hollweg \& Johnson 1988). Since \footnotetext{
$\begin{array}{ccccc}{ }^{1} \text { Space } & \text { Science Center and Department of } & \text { Physics, } \\ \text { University of New Hampshire, } & \text { Durham, NH } & \text { 03824; }\end{array}$ benjamin.chandran@unh.edu, s.bourouaine@unh.edu, phil.isenberg@unh.edu, daniel.verscharen@unh.edu

${ }^{2}$ Astronomy Department \& Theoretical Astrophysics Center, 601 Campbell Hall, The University of California, Berkeley, CA 94720; eliot@astro.berkeley.edu

${ }^{3}$ Harvard-Smithsonian Center for Astrophysics, Cambridge, MA 02138; jkasper@cfa.harvard.edu

${ }^{4}$ Merton College, University of Oxford, Oxford OX1 4JD, United Kingdom
}

there is abundant energy in non-compressive, large-scale, MHD-like fluctuations in the solar wind Belcher \& Davis 1971), such a cascade would lead to substantial energy in high-frequency $\mathrm{A} / \mathrm{IC}$ waves. Theoretical and numerical investigations, however, have shown that the energy of non-compressive MHD fluctuations cascades primarily to smaller $\lambda_{\perp}$ and only weakly to smaller $\lambda_{\|}$, where $\lambda_{\perp}$ and $\lambda_{\|}$are lengthscales perpendicular and parallel to the magnetic field $\boldsymbol{B}$ (Shebalin et al. 1983; Goldreich \& Sridhar 1995; Maron \& Goldreich 2001). Since the linear Alfvén wave frequency is $\sim v_{\mathrm{A}} / \lambda_{\|}$, where $v_{\mathrm{A}}$ is the Alfvén speed, the absence of an efficient cascade to small $\lambda_{\|}$implies that the cascade of energy from low frequency to high frequency is inefficient.

An alternative mechanism for generating high-frequency $\mathrm{A} / \mathrm{IC}$ waves is through a turbulent cascade involving compressive magnetosonic waves. The energy of fast magnetosonic waves ("fast waves") cascades radially in wavenumber space, from low frequency to high frequency (Cho \& Lazarian 2002). When the angle between the wavevector and the background magnetic field is small, high-frequency fast waves efficiently generate high-frequency Alfvén waves (Chandran 2005, 2008b). While these mechanisms could potentially be important in the corona ( $\mathrm{Li} \&$ Habbal 2001; Cranmer \& van Ballegooijen 2012), the observed anticorrelation between density fluctuations and magnetic-fieldstrength fluctuations in the solar wind at $1 \mathrm{AU}$ implies that fast waves comprise only a tiny fraction of the energy of the turbulence (Yao et al. 2011; Howes et al. 2012). This observational finding makes it unlikely that a fast-wave cascade can be a significant source of high-frequency fluctuations in the near-Earth solar wind. Another source of highfrequency A/IC waves is plasma instabilities (Kasper et al. 2002; Hellinger et al. 2006; Matteini et al. 2007; Bale et al. 2009; Wicks et al.2010). Instabilities driven by proton beams or alpha-particle beams could thermalize the beam energy and lead to substantial perpendicular ion heating (Gary et al. 2000; Hellinger \& Trávníček 2011; Verscharen \& Chandran 
2013). The extent to which beam-driven instabilities contribute to perpendicular ion heating in the solar wind remains an open question.

Dissipation of low-frequency turbulence, rather than highfrequency $\mathrm{A} / \mathrm{IC}$ waves, offers an alternative explanation for perpendicular ion heating in the solar wind. As mentioned previously, most of the fluctuation energy at small scales in solar-wind turbulence is believed to consist of non-compressive fluctuations with $\lambda_{\perp} \ll \lambda_{\|}$(Matthaeus et al. 1990; Bieber et al. 1994; Chen et al. 2012). If the dissipation of such fluctuations at small scales proceeded via linear wave damping, the result would be parallel ion heating and parallel electron heating rather than perpendicular ion heating (Quataert 1998). On the other hand, a number of studies have shown that the dissipation of lowfrequency turbulence via nonlinear mechanisms can violate $\mu$ conservation (Dmitruk et al. 2004; Parashar et al. 2009; Servidio et al. 2012). One such mechanism is stochastic heating (McChesnev et al.|1987; Karimabadi et al.|1994; Chen et al. 2001; Johnson \& Cheng 2001; Chaston et al. 2004; Fiksel et al. 2009; Bourouaine \& Chandran 2013). In stochastic ion heating, fluctuations at scales comparable to the ion gyroradii cause ion orbits to become disordered or stochastic in the plane perpendicular to $\boldsymbol{B}$, violating one of the preconditions for $\mu$ conservation (Kruskal 1962). The interactions of such ions with the time-varying electric field cause ions to diffuse in perpendicular kinetic energy, leading to perpendicular heating.

Recently, Kasper et al. (2013) (hereafter K13) presented detailed observations of the alpha-to-proton perpendicular temperature ratio $T_{\perp \alpha} / T_{\perp p}$ in the solar wind, and described how this temperature ratio depends upon the average flow velocity $U_{\alpha}$ of alpha particles in the proton frame as well as

$$
\beta_{\| \mathrm{p}}=\frac{8 \pi n_{\mathrm{p}} k_{\mathrm{B}} T_{\| \mathrm{p}}}{B_{0}^{2}} .
$$

They found that $T_{\perp \alpha} / T_{\perp \mathrm{p}}>6$ when $U_{\alpha} \ll v_{\mathrm{A}}$, that $T_{\perp \alpha} / T_{\perp \mathrm{p}}$ decreases to values of $\sim 4$ as $U_{\alpha} / v_{\mathrm{A}}$ increases towards unity when $\beta_{\| \mathrm{p}} \ll 1$, and that the decrease in $T_{\perp \alpha} / T_{\perp \mathrm{p}}$ with increasing $U_{\alpha} / v_{\mathrm{A}}$ is much less pronounced when $\beta_{\| \mathrm{p}} \gtrsim 1$. (These general trends are illustrated in Figure 5) K13 argued that these observations are consistent with Isenberg \& Vasquez's (2007) model of resonant cyclotron heating of heavy ions. In this paper, we address the question of whether these observations are consistent with stochastic ion heating by lowfrequency turbulence. Chandran et al. (2010) predicted that stochastic ion heating is less effective when the ions stream away from the Sun in the proton frame if the majority of the Alfvén-wave-like fluctuations propagate away from the Sun in the proton frame. This is qualitatively consistent with K13's observations. In this paper, we develop this idea in a more quantitative fashion to enable a better comparison between the stochastic-heating model and spacecraft observations. In Section 2, we extend the stochastic-heating theory developed by Chandran et al. (2010) to account for the motion of ions along $\boldsymbol{B}$. In Section 3 , we use these results to derive an analytic expression for the temperature ratios of different ion species in the solar wind. We then present theoretical calculations of how the alpha-to-proton temperature ratio depends upon $U_{\alpha}, \beta_{\| \mathrm{p}}$, and various properties of the turbulent fluctuations. We discuss our results and summarize our conclusions in Section 4

\section{STOCHASTIC HEATING AND PARALLEL ION MOTION}

We model the solar wind as a magnetized plasma containing low-frequency, quasi-2D turbulence. At perpendicular (parallel) lengthscales $\lambda_{\perp}\left(\lambda_{\|}\right)$satisfying $\rho_{\mathrm{p}} \ll \lambda_{\perp} \ll$ $L$, this turbulence has the properties of reduced magnetohydrodynamic (RMHD) turbulence, including the inequality $\lambda_{\perp} \ll \lambda_{\|}$, where $\rho_{\mathrm{p}}$ is the rms gyroradius of the full proton velocity distribution, and $L$ is the outer scale of the turbulence. We assume that the turbulence transitions to kinetic Alfvén wave (KAW) turbulence at lengthscales $\lesssim$ $\rho_{\mathrm{p}}$. By invoking the term "kinetic Alfvén wave," we do not mean to imply that the fluctuations oscillate monochromatically or that the turbulence is weak. Instead, we assume that the turbulence is strong and "critically balanced," in the sense that the linear and nonlinear operators in the governing equations are of comparable importance (Higdon 1984; Goldreich \& Sridhar 1995; Cho \& Lazarian 2004; Horbury et al. 2008; Schekochihin et al. 2009). The label "KAW" in the term "KAW turbulence" refers to the nature of these linear operators, whose eigenfunctions correspond to KAWs.

We now consider the stochastic heating of ions with mass $A m_{\mathrm{p}}$ and charge $Z e$, where $m_{\mathrm{p}}$ and $e$ are the proton mass and charge. We make no restriction on whether these ions are minor ions, alpha particles, or protons. For the moment, we focus on ions with velocity component $v_{\|}$parallel to the background magnetic field $\boldsymbol{B}_{0}$ and take $v_{\|}$to be approximately constant over the stochastic-heating timescale, where $v_{\|}$is measured in the average proton rest frame. Eventually, we will average over $v_{\|}$, but for now we define $Q_{\perp}\left(v_{\|}\right), T_{\perp}\left(v_{\|}\right)$, and $\rho\left(v_{\|}\right)$to be the stochastic heating rate, perpendicular temperature, and rms gyroradius of ions with parallel velocity $v_{\|}$, where $\rho\left(v_{\|}\right)=v_{\perp}\left(v_{\|}\right) / \Omega_{\mathrm{i}}$,

$$
v_{\perp}\left(v_{\|}\right)=\sqrt{\frac{2 k_{\mathrm{B}} T_{\perp}\left(v_{\|}\right)}{A m_{\mathrm{p}}}},
$$

and $\Omega_{\mathrm{i}}=Z e B_{0} /\left(A m_{\mathrm{p}} c\right)$ is the ion cyclotron frequency.

Although the ions may interact with turbulent fluctuations over a broad range of lengthscales, we only consider the contribution to $Q_{\perp}\left(v_{\|}\right)$from the electric and magnetic-field fluctuations at lengthscales $\sim \rho\left(v_{\|}\right)$. We define $\delta \boldsymbol{E}$ and $\delta \boldsymbol{B}$ to be the gyroscale electric and magnetic fields in the average proton rest frame. The quantity

$$
\delta \boldsymbol{v}=c \frac{\delta \boldsymbol{E} \times \boldsymbol{B}}{B^{2}}
$$

is then the gyroscale $\boldsymbol{E} \times \boldsymbol{B}$ velocity in the average proton frame. We assume that $\delta B \ll B_{0}$. Neglecting the component of $\delta \boldsymbol{E}$ parallel to the magnetic field and corrections of order $\delta B / B_{0}$, we rewrite Equation (3) in the form $\delta \boldsymbol{E}=$ $-\delta \boldsymbol{v} \times \boldsymbol{B}_{0} / c$. We define the Elsässer variables in the average proton rest frame as

$$
\boldsymbol{z}^{ \pm}=\delta \boldsymbol{v} \mp v_{\mathrm{A}} \frac{\delta \boldsymbol{B}_{\perp}}{B_{0}}
$$

where $\delta \boldsymbol{B}_{\perp}=\delta \boldsymbol{B}-\hat{\boldsymbol{b}}(\hat{\boldsymbol{b}} \cdot \delta \boldsymbol{B}), \quad \hat{\boldsymbol{b}}=\boldsymbol{B}_{0} / \boldsymbol{B}_{0}, \quad v_{\mathrm{A}}=$ $B_{0} / \sqrt{4 \pi n_{\mathrm{p}} m_{\mathrm{p}}}$ is the (proton) Alfvén speed, and $n_{\mathrm{p}}$ is the proton number density. 
We define the " $v_{\|}$frame" to be the reference frame moving at velocity $v_{\|} \hat{\boldsymbol{b}}_{0}$ with respect to the average proton rest frame. The gyroscale electric-field fluctuation in this frame is given by $\delta \boldsymbol{E}^{\prime}=\delta \boldsymbol{E}+v_{\|} \hat{\boldsymbol{b}} \times \delta \boldsymbol{B} / c$. We take $\left|v_{\|}\right|$and $v_{\mathrm{A}}$ to be $\ll c$ and thus neglect the difference between $\boldsymbol{B}$ in the $v_{\|}$ frame and $\boldsymbol{B}$ in the proton frame. The $\boldsymbol{E} \times \boldsymbol{B}$ velocity in the $v_{\|}$frame is then given by $\delta \boldsymbol{v}^{\prime}=c \delta \boldsymbol{E}^{\prime} \times \boldsymbol{B} / B^{2}$. Neglecting corrections of order $\delta B / B_{0}$, we obtain

$$
\delta \boldsymbol{v}^{\prime}\left(v_{\|}\right)=\frac{\boldsymbol{z}^{+}}{2}\left(1-\frac{v_{\|}}{v_{\mathrm{A}}}\right)+\frac{\boldsymbol{z}^{-}}{2}\left(1+\frac{v_{\|}}{v_{\mathrm{A}}}\right) .
$$

The stochastic heating rate per unit mass $Q_{\perp}\left(v_{\|}\right)$can now be obtained in exactly the same way as in the phenomenological treatment of Chandran et al. (2010), but replacing their $\delta v_{\mathrm{i}}$ (the rms value of $\delta \boldsymbol{v}$ ) with $\delta v_{\text {rms }}^{\prime}$, the rms value of $\delta \boldsymbol{v}^{\prime}$. This leads to the expression

$$
Q_{\perp}\left(v_{\|}\right)=\frac{c_{1}\left[\delta v_{\mathrm{rms}}^{\prime}\left(v_{\|}\right)\right]^{3}}{\rho\left(v_{\|}\right)} \exp \left(-\frac{c_{2}}{\varepsilon}\right),
$$

where

$$
\varepsilon=\frac{\delta v_{\mathrm{rms}}^{\prime}\left(v_{\|}\right)}{v_{\perp}\left(v_{\|}\right)} .
$$

The quantities $c_{1}$ and $c_{2}$ are dimensionless constants of order unity. The derivation of Equation (6) assumes that the ion thermal speeds are $\lesssim v_{\mathrm{A}}$. Assuming that the ion temperature anisotropies are not very large and that the ion thermal speed is comparable to the proton thermal speed, this condition is approximately equivalent to

$$
\beta_{\| \mathrm{p}} \lesssim 1
$$

We return to this condition in Section 3 .

We define the gyroscale fractional cross helicity

$$
\sigma=\frac{\left\langle\left|z^{+}\right|^{2}-\left|z^{-}\right|^{2}\right\rangle}{\left\langle\left|z^{+}\right|^{2}+\left|z^{-}\right|^{2}\right\rangle}
$$

the gyroscale Alfvén ratio

$$
r_{\mathrm{A}}=\left(\frac{B_{0}}{v_{\mathrm{A}}}\right)^{2} \frac{\left\langle|\delta \boldsymbol{v}|^{2}\right\rangle}{\left\langle\left|\delta \boldsymbol{B}_{\perp}\right|^{2}\right\rangle}
$$

and the quantity

$$
W=\frac{1}{4}\left\langle\left|z^{+}\right|^{2}+\left|z^{-}\right|^{2}\right\rangle,
$$

where $\langle\ldots\rangle$ indicates a time or volume average. If $\rho\left(v_{\|}\right) \gg$ $\rho_{\mathrm{p}}$, then the gyroscale fluctuations are in the RMHD regime, $\delta \boldsymbol{B}_{\perp}=\delta \boldsymbol{B}$ to a good approximation, and the $\boldsymbol{E} \times \boldsymbol{B}$ velocity is approximately equal to the component of the average proton velocity perpendicular to $\boldsymbol{B}$. In this case, $W$ is the energy of the gyroscale fluctuations per unit mass. On the other hand, if $\rho\left(v_{\|}\right) \simeq \rho_{\mathrm{p}}$, then the fluctuations are at the transition to the KAW regime, $\hat{b} \cdot \delta B \sim \delta B_{\perp}$, the protons do not move at the $\boldsymbol{E} \times \boldsymbol{B}$ velocity, and $W$ differs (by a factor of order unity) from the energy per unit mass (Hollweg 1999). Upon taking the rms of the right-hand side of Equation (5), we obtain

$$
\delta v_{\text {rms }}^{\prime}\left(v_{\|}\right)=(\chi W)^{1 / 2},
$$

where

$$
\chi=\frac{2}{r_{\mathrm{A}}+1}\left(r_{\mathrm{A}}+\frac{v_{\|}^{2}}{v_{\mathrm{A}}^{2}}\right)-\frac{2 \sigma v_{\|}}{v_{\mathrm{A}}} .
$$

\section{ION TEMPERATURE RATIOS IN THE SOLAR WIND}

In the solar wind at heliocentric distances $r$ between $0.3 \mathrm{AU}$ and $1 \mathrm{AU}$, the perpendicular temperatures of protons and alpha particles decrease with increasing $r$, but not as fast as they would decrease in the case of adiabatic expansion (Marsch et al. 1982a, b; Hellinger et al. 2013). This implies that there is a competition between adiabatic cooling and heating and that the heating timescale $t_{\mathrm{h}}$ is of the same order of magnitude as the cooling or expansion timescale $t_{\exp }=$ $r /\left(U+v_{\|}\right)$, where $U$ is the proton outflow velocity. We assume that this is true not just for protons and alpha particles, but for all ion species. We further assume that the dominant perpendicular ion heating mechanism is stochastic heating, so that $t_{\mathrm{h}} \simeq v_{\perp i}^{2} / Q_{\perp}$, where we have suppressed the dependence of these quantities on $v_{\|}$for brevity. Approximately equating $t_{\mathrm{h}}$ and $t_{\mathrm{exp}}$ leads to the condition

$$
\varepsilon^{-3} \exp \left(\frac{c_{2}}{\varepsilon}\right) \simeq \frac{c_{1} r \Omega_{\mathrm{i}}}{U+v_{\|}} .
$$

A version of this relation with $c_{1} \simeq 1$ and $v_{\|}=0$ was previously obtained by Chandran (2010) in a study of ion temperatures in coronal holes. Because the left-hand side of Equation (14) is a rapidly varying function of $\varepsilon$, Equation (14) leads to similar values of $\varepsilon$ for different ion species. We illustrate this point in Figure 1, which plots the solution of Equation (14) for plasma parameters characteristic of the slow solar wind near Earth using the values $c_{1}=0.74$ and $c_{2}=0.21 \mathrm{ob}$ tained in a recent numerical simulation of the stochastic heating of test-particles in RMHD turbulence (Xia et al. 2013). When Equation (14) is applied to ions of the same species in the solar wind with different values of $v_{\|}$, these variations in $v_{\|}$lead to fractional variations in the right-hand side of Equation (14) that are small, because the ion thermal speeds are $\ll U$. Figure 1 shows that small fractional variations in the right-hand side of Equation (14) lead to extremely small variations in $\varepsilon$. In the analysis to follow, we make the approximation that $\varepsilon$ is independent of both $v_{\|}$and $Z / A$.

To illustrate how ions can achieve the value of $\varepsilon$ needed to satisfy Equation (14), we consider the hypothetical evolution of ions that are initially sufficiently cool that $\varepsilon$ is larger than the value in Equation (14). For such ions, $t_{\mathrm{h}}$ is initially smaller than $t_{\text {exp }}$, and thus heating initially dominates over cooling, causing $T_{\perp \mathrm{i}}$ to increase. In the case of minor ions, this heating draws a negligible amount of power from the turbulence, and $T_{\perp}\left(v_{\|}\right)$simply increases until $\varepsilon$ decreases to the value in Equation (14). Stochastic heating cannot increase $T_{\perp}\left(v_{\|}\right)$any further, because a higher $T_{\perp}\left(v_{\|}\right)$would imply a smaller value of $\varepsilon$ and hence an exponentially smaller value of $Q_{\perp}\left(v_{\|}\right)$. In the case of protons and alpha particles, stochastic ion heating can drain a significant fraction of the turbulent cascade power, thereby reducing $\delta v^{\prime}$. For these ions, Equation (14) is in general satisfied by some combination of heating of the ions and damping of the gyroscale fluctuations.

We now estimate $T_{\perp \mathrm{i}} / T_{\perp \mathrm{p}}$, the ion-to-proton perpendicular temperature ratio for ion species $i$, which we take to have 


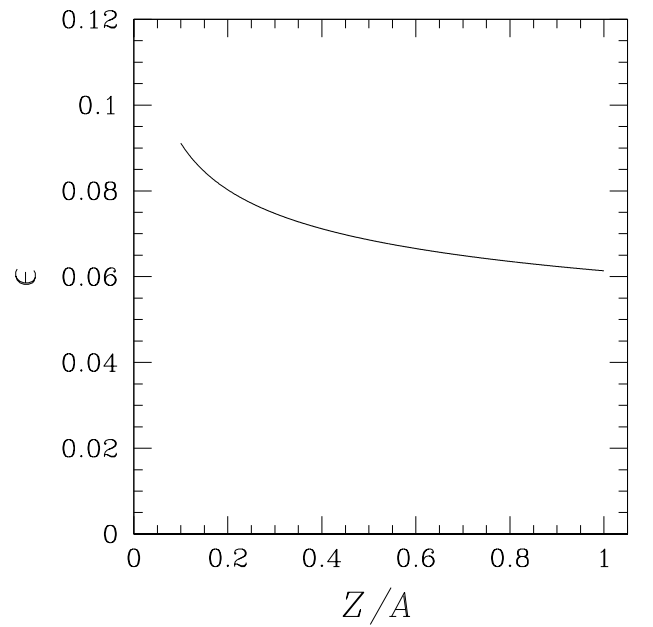

FIG. 1. - Stochasticity parameter $\varepsilon=\delta v_{\text {rms }}^{\prime}\left(v_{\|}\right) / v_{\perp}\left(v_{\|}\right)$as a function of $Z / A$, which is the ion charge-to-mass ratio in units of the proton chargeto-mass ratio. This value of $\varepsilon$ is obtained by solving the ion energy balance equation, Equation (14), for the case in which $c_{1}=0.74, c_{2}=0.21, B=5 \mathrm{nT}$, $U+v_{\|}=400 \mathrm{~km} / \mathrm{s}$, and $r=1 \mathrm{AU}$. The weak variation of $\varepsilon$ with $Z / A$ justifies our approximation that $\varepsilon$ is the same for all ions at the same location.

mass $A m_{\mathrm{p}}$ and charge $Z e$, as above. We do not attempt to predict the way that proton and alpha-particle heating alter the turbulent power spectrum at wavenumbers $\sim \rho_{\mathrm{p}}^{-1}$. Instead, we parametrize the dependence of $W$ on $\rho\left(v_{\|}\right)$through the equation

$$
W=W_{\mathrm{p}}\left[\frac{\rho\left(v_{\|}\right)}{\rho_{\mathrm{p}}}\right]^{2 a},
$$

where the constant $a$ is a free parameter, and $W_{\mathrm{p}}$ is a constant whose value has no influence on our estimate of $T_{\perp \mathrm{i}} / T_{\perp \mathrm{p}}$. If $\rho\left(v_{\|}\right)$and $\rho_{\mathrm{p}}$ were both in the inertial range of solar-wind turbulence, and if the total energy spectrum were $\propto k_{\perp}^{-3 / 2}$ in the inertial range, then $a$ would be $1 / 4$. However, we assume that $\rho\left(v_{\|}\right)$is in the range of $\rho_{p}$ to a few $\rho_{p}$. At perpendicular wavenumbers $\sim \rho_{\mathrm{p}}^{-1}$, there are two effects that influence the value of $a$. First, dissipation acts to steepen the power spectra of $\boldsymbol{E}$ and $\boldsymbol{B}$ fluctuations, thereby acting to increase $a$. Second, the cross-field motions of electrons and protons are only partially coupled. This decoupling causes the electric-field (magnetic-field) power spectrum to flatten (steepen) at $k_{\perp} \gtrsim \rho_{\mathrm{p}}^{-1}$ (Bale et al. 2005; Howes et al. 2008; Schekochihin et al. 2009). Because $W$ contains contributions from both $\boldsymbol{E} \times \boldsymbol{B}$ velocity fluctuations and $\boldsymbol{B}$ fluctuations, we conjecture that the decoupling of electrons and protons at scales $\sim \rho_{\mathrm{p}}$ acts to reduce $a$ slightly relative to the value that would apply at inertial-range scales. We do not attempt to take these various effects into account and determine the most accurate or physically reasonable value of $a$. Instead, we consider a few different values as numerical examples, focusing on the case in which $a=1 / 4$. To further simplify the analysis, we treat $r_{\mathrm{A}}$ and $\sigma$ as constants, thereby neglecting any possible variation in the Alfvén ratio and fractional cross helicity over scales between $\sim \rho_{p}$ and $\rho\left(v_{\|}\right)$.

Using Equation (12), we re-write Equation (7) in the form

$$
\left[v_{\perp \mathrm{i}}\left(v_{\|}\right)\right]^{2}=\varepsilon^{-2 \eta} W_{\mathrm{p}}^{\eta}\left(\frac{A}{Z w_{\perp \mathrm{p}}}\right)^{2 a \eta} \chi^{\eta}
$$

where $w_{\perp \mathrm{p}}$ is the perpendicular thermal speed of the full proton distribution, and

$$
\eta=\frac{1}{1-a}
$$

Upon averaging Equation (16) over $v_{\|}$and making the approximation that $\varepsilon$ is the same for all values of $v_{\|}$, we obtain

$$
w_{\perp \mathrm{i}}^{2}=\varepsilon^{-2 \eta} W_{\mathrm{p}}^{\eta}\left(\frac{A}{Z w_{\perp \mathrm{p}}}\right)^{2 a \eta}\left\langle\chi^{\eta}\right\rangle_{\mathrm{i}},
$$

where $\langle\ldots\rangle_{i}$ indicates an average over the $v_{\|}$distribution of species $\mathrm{i}, w_{\perp \mathrm{i}}^{2}=\left\langle\left[v_{\perp \mathrm{i}}\left(v_{\|}\right)\right]^{2}\right\rangle_{\mathrm{i}}$, and the subscripts $\mathrm{i}=\mathrm{p}$ and $\mathrm{i}=\alpha$ correspond to protons and alpha particles, respectively. Applying Equation (18) to the protons, we obtain

$$
w_{\perp p}^{2}=\varepsilon^{-2} W_{\mathrm{p}}\left\langle\chi^{\eta}\right\rangle_{\mathrm{p}}^{1 / \eta}
$$

Using Equation (19) to eliminate $W_{\mathrm{p}}$ in Equation (18), we find that

$$
\frac{T_{\perp \mathrm{i}}}{T_{\perp \mathrm{p}}}=A\left(\frac{A}{Z}\right)^{2 a \eta} \frac{\left\langle\chi^{\eta}\right\rangle_{\mathrm{i}}}{\left\langle\chi^{\eta}\right\rangle_{\mathrm{p}}}
$$

where $T_{\perp \mathrm{i}}$ and $T_{\perp \mathrm{p}}$ (without the functional dependence on $v_{\|}$) are the perpendicular temperatures of the full ion and proton distributions. We take the $v_{\|}$distribution of each ion species to be a shifted Maxwellian with average parallel velocity $U_{\mathrm{i}}$ (where $U_{\mathrm{p}}=0$ ), parallel temperature $T_{\| \mathrm{i}}$, and parallel thermal speed defined by $w_{\| \mathrm{i}}=\sqrt{2 k_{\mathrm{B}} T_{\| \mathrm{i}} /\left(A m_{\mathrm{p}}\right)}$. Thus,

$$
\left\langle\chi^{\eta}\right\rangle_{\mathrm{i}}=\frac{1}{\sqrt{\pi} w_{\| \mathrm{i}}} \int_{-\infty}^{\infty} d v_{\|} \chi^{\eta} \exp \left(-\frac{\left(v_{\|}-U_{\mathrm{i}}\right)^{2}}{w_{\| \mathrm{i}}^{2}}\right) .
$$

We now use Equation (20) to calculate the alpha-to-proton perpendicular temperature ratio. In Figure 2, we illustrate how $T_{\perp \alpha} / T_{\perp p}$ in our model depends upon $U_{\alpha}, \sigma, r_{\mathrm{A}}, a$, and $\beta_{\| p}$ for the case in which

$$
T_{\| \alpha}=5.2 T_{\| \mathrm{p}}
$$

which corresponds to the average parallel temperature ratio measured by the Wind spacecraft in the weakly collisional solar-wind streams examined by Kasper et al. (2008). As shown in the upper-left panel of Figure 2, if $\sigma$ is close to one, $\beta_{\| \mathrm{p}}=0.1$, and $r_{\mathrm{A}}=1.5$, then $T_{\perp \alpha} / T_{\perp \mathrm{p}}$ undergoes a marked decrease as $U_{\alpha} / v_{\mathrm{A}}$ increases from zero to one, because the transformation from the average proton frame to the average alpha-particle frame reduces the amplitude of the electric field fluctuations, which are the source of the heating. One way of viewing this is through the expression for the gyroscale $\boldsymbol{E} \times \boldsymbol{B}$ velocity $\delta v^{\prime}$ in the $v_{\|}$frame given in Equation (5). As $v_{\|} / v_{\mathrm{A}}$ increases from 0 to 1 , the contribution of the antisunward-propagating $z^{+}$fluctuations to $\delta v^{\prime}$ decreases, and the contribution to $\delta v^{\prime}$ from the sunward-propagating $z^{-}$fluctuations increases. When $\sigma$ is close to 1 , the dominant effect is the reduction in the contribution from the $z^{+}$fluctuations. In contrast, as $\sigma$ decreases to zero, the increase in the contribution to $\delta v^{\prime}$ from $z^{-}$becomes increasingly important and can even cause $\delta v_{\text {rms }}^{\prime}$ to increase as $v_{\|}$increases towards $v_{\mathrm{A}}$.

The upper-right panel of Figure 2 shows how the alpha-toproton temperature ratio depends upon $U_{\alpha}$ for different values of $\beta_{\| p}$. When $\beta_{\| p}$ is small, the bulk of the alpha particle distribution can be taken to have velocities $\simeq U_{\alpha}$. Thus, as $U_{\alpha}$ 

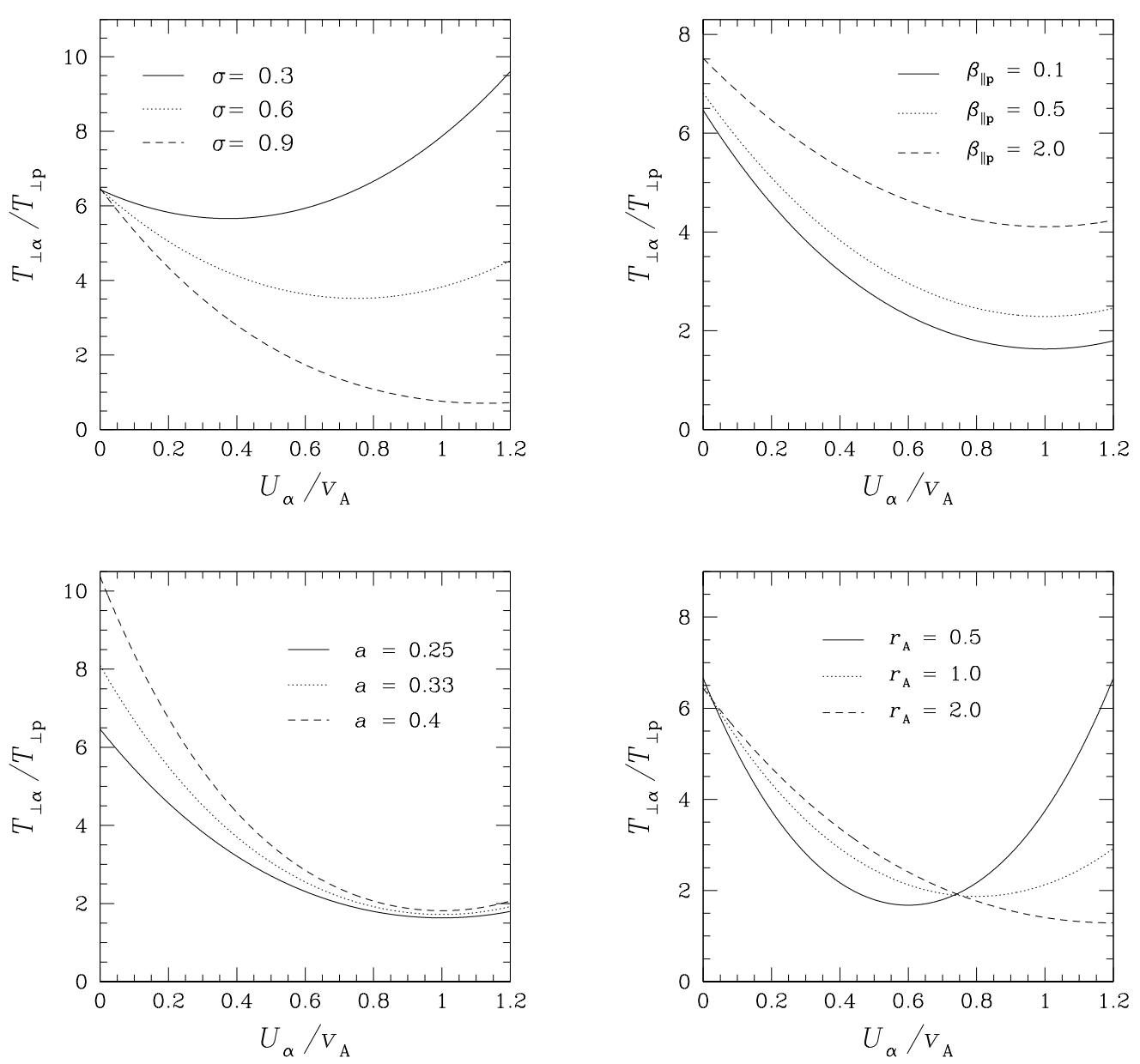

FIG. 2.- Dependence of the alpha-to-proton perpendicular temperature ratio on the alpha-proton drift velocity $U_{\alpha}$, the gyroscale fractional cross helicity $\sigma$, the value of $\beta_{\| \mathrm{p}}$, the scaling exponent $a$ of the rms fluctuation amplitude at scales $\sim \rho_{\mathrm{p}}$, and the gyroscale Alfvén ratio $r_{\mathrm{A}}$. For each plot, $T_{\| \alpha}=5.2 T_{\| \mathrm{p}}$. Except where indicated otherwise, these figures make use of the fiducial parameter values $\sigma=0.8, \beta_{\| \mathrm{p}}=0.1, a=0.25$, and $r_{\mathrm{A}}=1.5$.

increases from 0 to $v_{\mathrm{A}}$, the electric field fluctuations seen by the alphas weaken considerably when $\sigma$ is close to one, causing $T_{\perp \alpha} / T_{\perp \mathrm{p}}$ to decrease. On the other hand, if $\beta_{\| \mathrm{p}} \gtrsim 1$, then the parallel thermal speed exceeds $v_{\mathrm{A}}$, the particles are never localized within a narrow band of $v_{\|} / v_{\mathrm{A}}$ values, and there is thus less of a reduction in $T_{\perp \alpha} / T_{\perp \mathrm{p}}$ as $U_{\alpha} / v_{\mathrm{A}}$ increases from 0 to 1 . Also, when $\beta_{\| \mathrm{p}} \gtrsim 1$ and $T_{\| \alpha}>4 T_{\| \mathrm{p}}$, alpha particles are more likely than protons to satisfy either $v_{\|}<0$ or $v_{\|}>v_{\mathrm{A}}$, which enhances $T_{\perp \alpha} / T_{\perp \mathrm{p}}$ relative to the low- $\beta_{\| \mathrm{p}}$ case at all values of $U_{\alpha} / v_{\mathrm{A}}$.

The lower-left panel of Figure 2 shows how the alpha-toproton temperature ratio depends upon $U_{\alpha}$ for different values of $a$. Larger values of $a$ correspond to a steeper turbulent power spectrum at wavenumbers of order $\rho_{\mathrm{p}}^{-1}$. Because the alpha-particle gyroradii are larger than $\rho_{p}$ in the solar wind, increasing $a$ increases the amplitudes of the fluctuations that heat the alpha particles relative to the amplitudes of the fluctuations that heat the protons, thereby increasing $T_{\perp \alpha} / T_{\perp p}$. This effect weakens in the lower-left panel of Figure 2 as $U_{\alpha} / v_{\mathrm{A}} \rightarrow 1$ because $T_{\perp \alpha} / T_{\perp \mathrm{p}}$ decreases, thereby reducing the alpha-particle gyroradii.

The lower-right panel of Figure 2 shows how the alpha-toproton temperature ratio depends upon $U_{\alpha}$ for different values of $r_{\mathrm{A}}$. The magnetic-field fluctuation in the average proton rest frame, $\boldsymbol{\delta} \boldsymbol{B}$, does not contribute to particle energization in the average proton rest frame, since the time derivative of the particle kinetic energy is simply Zev $\cdot \delta \boldsymbol{E}$. However, the electric field in the $v_{\|}$frame is $\delta \boldsymbol{E}+\Delta \boldsymbol{E}$, where $\Delta \boldsymbol{E}=v_{\|} \hat{\boldsymbol{b}} \times$ $\delta B / c$, and thus $\delta B$ can contribute to the heating rate in the $v_{\|}$frame through the action of $\Delta \boldsymbol{E}$. The gyroscale Alfvén ratio $r_{\mathrm{A}}$ controls the relative contributions of $\Delta \boldsymbol{E}$ and $\delta \boldsymbol{E}$ to the stochastic heating rate. As $r_{\mathrm{A}}$ is decreased, $\Delta \boldsymbol{E}$ becomes larger relative to $\delta \boldsymbol{E}$, which can lead to an increase in the total stochastic heating rate as $v_{\|} / v_{\mathrm{A}}$ is increased to values $\sim 1$. On the other hand, as $r_{\mathrm{A}}$ is increased, the contribution of $\Delta \boldsymbol{E}$ to particle heating becomes less important, and the stochastic heating rate undergoes a smaller increase as $v_{\|}$is increased towards $v_{\mathrm{A}}$.

In Figure 3, we plot $T_{\perp \alpha} / T_{\perp p}$ as a function of $U_{\alpha} / v_{\mathrm{A}}$ and $\beta_{\| \mathrm{p}}$ for nine different combinations of the parameters $r_{\mathrm{A}}$ and $\sigma$. For all panels of this figure, we set $T_{\| \alpha} / T_{\| \mathrm{p}}=5.2$, as in Equation (22), and $a=0.25$. The portion of each plot with $U_{\alpha}<0$ is obtained by reflecting the upper half of the plot through the line $U_{\alpha}=0$. The lower half of each plot represents cases in which $\boldsymbol{B}_{0}$ is directed towards the Sun, so that $U_{\alpha}$ and $\sigma$ are both negative. Thus, in the upper-right panel of Figure 3 with the label $\sigma= \pm 0.4$, the value $\sigma=+0.4$ corresponds to the upper half of the plot in which $U_{\alpha}>0$, and the 

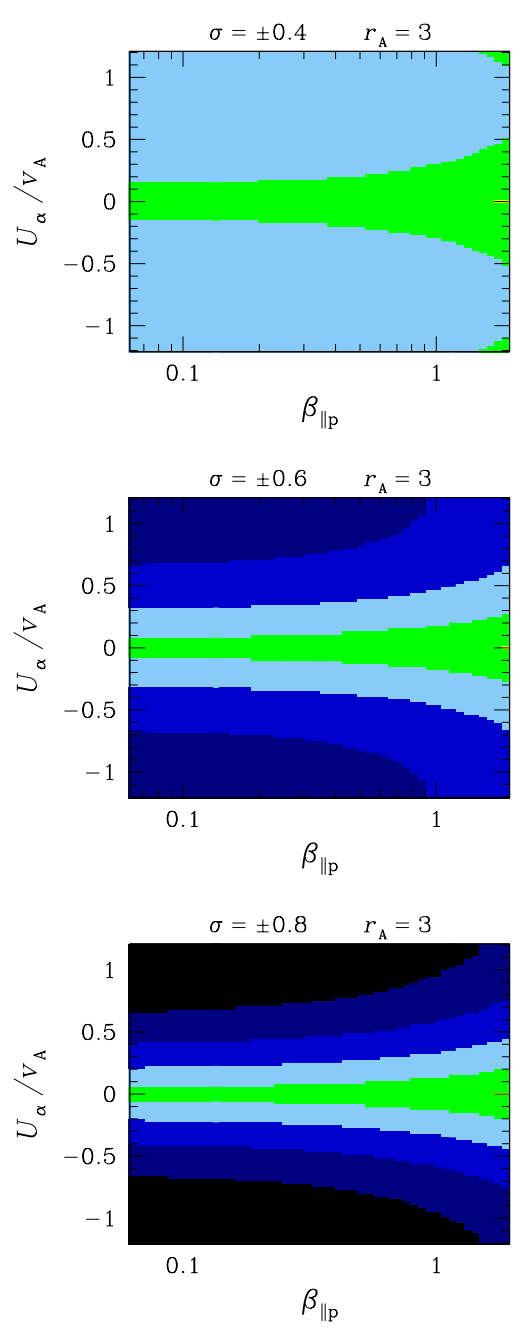
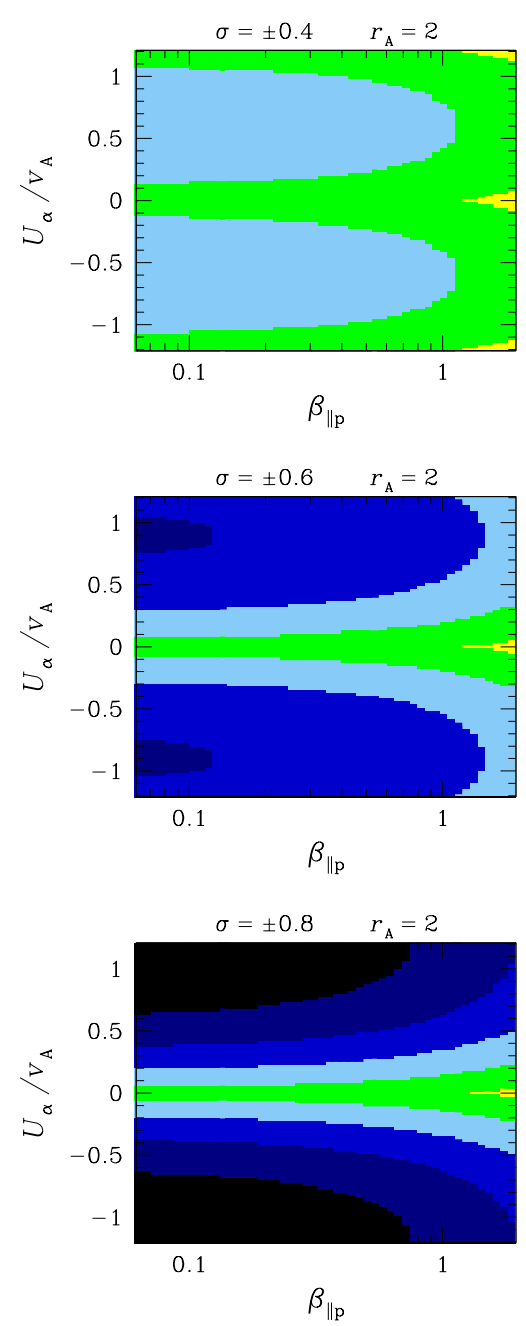
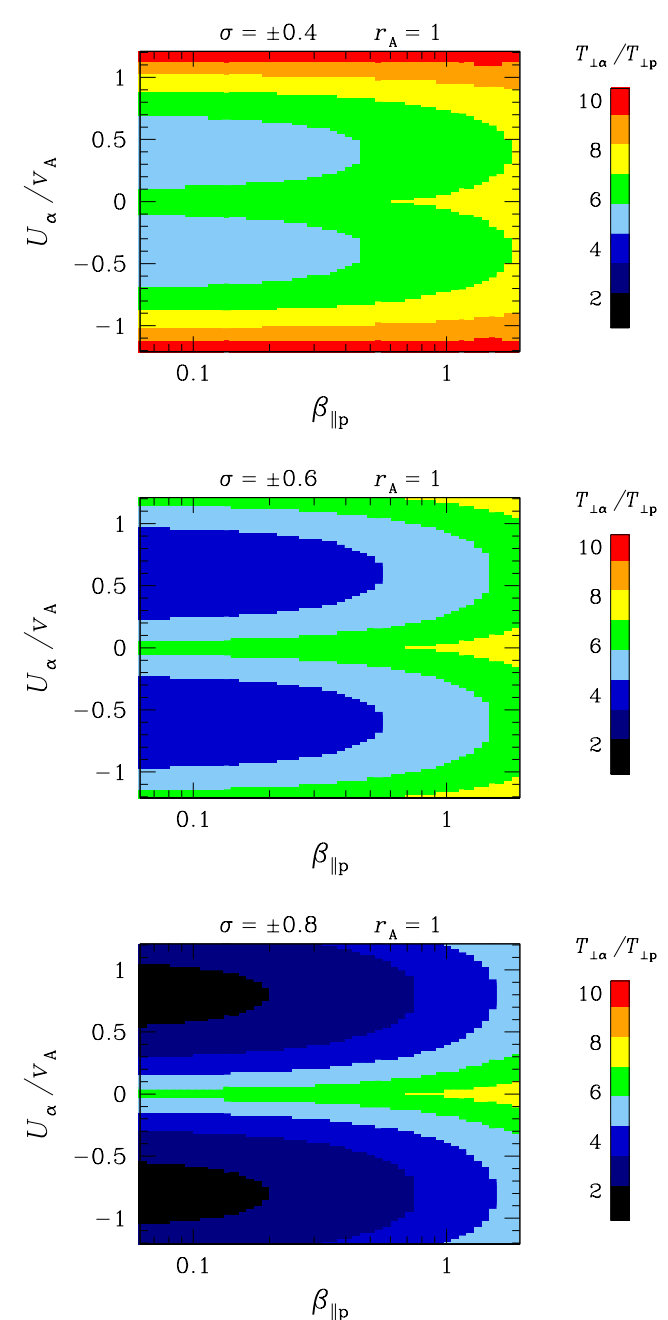

FIG. 3. - Color-scale plots of $T_{\perp \alpha} / T_{\perp p}$ for different choices of the gyroscale fractional cross helicity $\sigma$ and gyroscale Alfvén ratio $r_{\mathrm{A}}$. For all figures, $a=0.25$ and $T_{\| \alpha} / T_{\| \mathrm{p}}=5.2$. These results can be compared directly to the Wind measurements in Figure 2 of Kasper et al. (2013).

value $\sigma=-0.4$ corresponds to the lower half of the plot in which $U_{\alpha}<0$. These plots can be compared to K13's Figure 2, which plots, in the same coordinate plane, the average value of $T_{\perp \alpha} / T_{\perp \mathrm{p}}$ in measurements of weakly collisional solar-wind streams from the Wind spacecraft. However, because of Equation (8), we have limited our plots to $\beta_{\| p} \leq 2$, whereas K13's Figure 2 includes larger $\beta_{\| p}$ values.

All of the panels in Figure 3 share three features with the Wind data plotted in K13's Figure 2 . First, $6 \lesssim T_{\perp \alpha} / T_{\perp \mathrm{p}} \lesssim 7$ within a band of small- $U_{\alpha} / v_{\mathrm{A}}$ values when $\beta_{\| \mathrm{p}} \lesssim 1$. Second, $T_{\perp \alpha} / T_{\perp \mathrm{p}}$ decreases with increasing $U_{\alpha} / v_{\mathrm{A}}$ at small- $\beta_{\| \mathrm{p}}$, because the electric field seen by the alpha particles is reduced when the alpha-particles drift in the same direction that the majority of the RMHD fluctuations propagate. Third, this decrease is less pronounced at $\beta_{\| \mathrm{p}} \sim 1$, in part because the increase in $w_{\| \alpha} / v_{\mathrm{A}}$ means that the averaging over $v_{\|}$in Equation (18) increasingly smoothes out the vertical variations in each panel, and in part because a larger value of $w_{\| \alpha} / v_{\mathrm{A}}$ means that more alpha particles satisfy $v_{\|}<0$ or $v_{\|}>v_{\mathrm{A}}$, either of which conditions enhances the electric field in the $v_{\|}$frame. On the other hand, several of the panels in Figure 3 exhibit enhanced $T_{\perp \alpha} / T_{\perp p}$ values at $U_{\alpha} \simeq v_{\mathrm{A}}$, which are not seen in the Wind data. This discrepancy becomes increas- ingly pronounced as $|\sigma|$ and/or $r_{\mathrm{A}}$ decrease. The ability of our model to explain the Wind data thus depends on the values of $\sigma$ and $r_{\mathrm{A}}$ in the solar wind, as we discuss further in Section 4

In Figure 4 we illustrate how the color-scale plots of Figure 3 change when we use a larger value of $a$ or a smaller value of $T_{\| \alpha} / T_{\| \mathrm{p}}$. The left panel of Figure 4 is the same as the middle plot in Figure 3. except that $a$ has been increased from 0.25 to 0.35 . This increase in $a$ increases $T_{\perp \alpha} / T_{\perp p}$ at all locations in this plot because of the change in the factor of $(A / Z)^{2 a \eta}$ on the right-hand side of Equation (20). The right panel of Figure 4 is the same as the left panel of Figure 4, except that $T_{\| \alpha} / T_{\| \mathrm{p}}$ has been decreased from 5.2 to 4 . The primary effect of decreasing $T_{\| \alpha} / T_{\| p}$ is to shift the plot towards the right. This is because the averaging over $v_{\|}$that occurs in Equation (20) smoothes out the vertical variations in $T_{\perp \alpha} / T_{\perp \mathrm{p}}$ in these plots when $w_{\| \alpha} \gtrsim v_{\mathrm{A}}$, and the condition $w_{\| \alpha}=v_{\mathrm{A}}$ is satisfied at larger $\beta_{\| \mathrm{p}}$ when $T_{\| \alpha} / T_{\| \mathrm{p}}$ is smaller.

In Figure 5, we average $T_{\perp \alpha} / T_{\perp p}$ over uniform distributions of $|\sigma|$ and $r_{\mathrm{A}}$. In particular, we calculate $T_{\perp \alpha} / T_{\perp \mathrm{p}}$ for each of ten values of $|\sigma|$ evenly spaced between 0.4 and 0.8 (inclusive) and each of ten values of $r_{\mathrm{A}}$ evenly spaced between 1.5 and 2.5 (inclusive), and then we average the resulting 100 values of $T_{\perp \alpha} / T_{\perp \mathrm{p}}$. For this figure, we set $a=0.25$ and $T_{\| \alpha}=5.2 T_{\| \mathrm{p}}$. Of all the plots in Figures 3 through 5 . Figure 5 is the most 

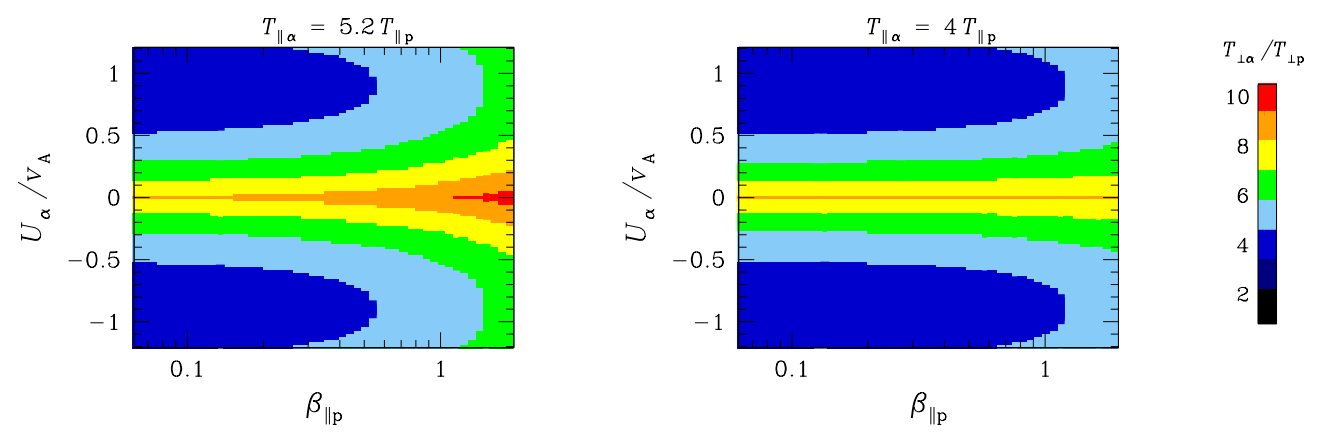

FIG. 4.- Color-scale plots of $T_{\perp \alpha} / T_{\perp \mathrm{p}}$ as a function of $\beta_{\| \mathrm{p}}$ and the average alpha-particle velocity $U_{\alpha}$. In both panels, the scaling exponent $a$ is 0.35 , and the gyroscale Alfvén ratio $r_{\mathrm{A}}$ is 2 . The gyroscale fractional cross helicity $\sigma$ is 0.6 in the upper half planes in which $U_{\alpha}>0$ and -0.6 in the lower half planes in which $U_{\alpha}<0$. The parallel temperature ratio $T_{\| \alpha} / T_{\| \mathrm{p}}$ is 5.2 in the left panel and 4.0 in the right panel. The color bar in this figure is the same as in Figure 3 to facilitate comparison of these figures, but the two darkest colors are not used in either panel of Figure 4

similar to K13's Figure 2.

We note that $\mathrm{K} 13$ found enhanced values of $T_{\perp \mathrm{p}} / T_{\| \mathrm{p}}$ in the upper-left and lower-left corners of the plane shown in each of the plots of Figures 3 through 5 . They interpreted this result within the context of a resonant-cyclotron-heating model as evidence that when $T_{\perp \alpha} / T_{\perp p}$ is comparatively small (i.e., $\sim 4$ rather than $\sim 7$ ), the alpha-particles drain less power from the $\mathrm{A} / \mathrm{IC}$ waves, enabling the $\mathrm{A} / \mathrm{IC}$ waves to cause enhanced perpendicular proton heating. It is possible that a similar interpretation can be applied within the context of a stochasticheating model. In this case, weaker alpha-particle heating would drain less power from the turbulent cascade, enabling more energy to cascade to the proton-gyroradius scale and enhancing the stochastic heating of protons. However, a direct prediction of the magnitude of this effect is beyond the scope of this paper.

\section{DISCUSSION AND CONCLUSION}

In this paper, we extend Chandran et al's (2010) theoretical treatment of stochastic heating to account for the motion of ions along the background magnetic field. Using this more general theory, we derive an analytic expression for the ionto-proton perpendicular temperature ratio $T_{\perp \mathrm{i}} / T_{\perp \mathrm{p}}$ in the solar wind under the assumption that stochastic heating is the

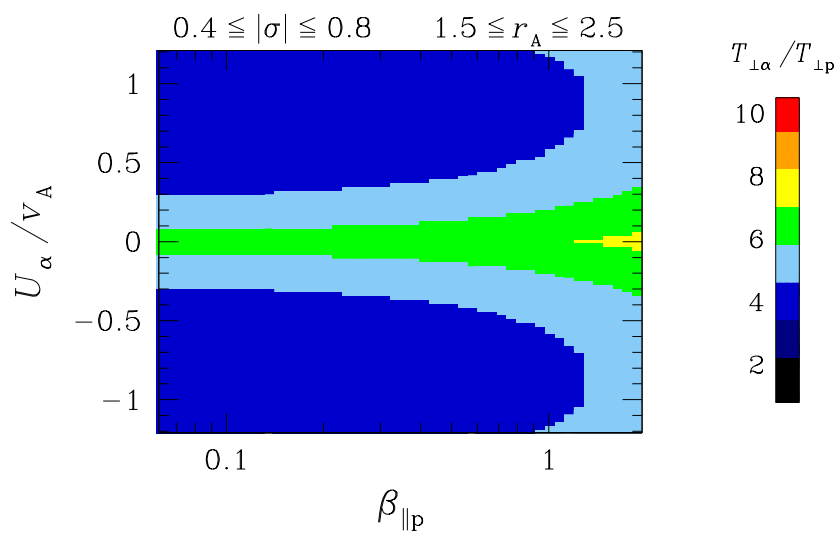

FIG. 5.- Color-scale plot of $T_{\perp \alpha} / T_{\perp \mathrm{p}}$ as a function of $\beta_{\| \mathrm{p}}$ and $U_{\alpha}$ averaging over uniform distributions of $|\sigma|$ and $r_{\mathrm{A}}$, which vary throughout the ranges $0.4 \leq|\sigma| \leq 0.8$ and $1.5 \leq r_{\mathrm{A}} \leq 2.5$. The parallel temperature ratio $T_{\| \alpha} / T_{\| \mathrm{p}}$ is assumed to be 5.2, and $a=0.25$. The color bar is the same as in Figures 3 and 4t to facilitate comparison with these figures, but several colors that appear in this color bar are not needed for this plot. dominant perpendicular heating mechanism for all ions. This expression determines the dependence of $T_{\perp \mathrm{i}} / T_{\perp \mathrm{p}}$ on the average drift velocity of the ions of species i relative to the protons, the parallel thermal speeds of both species, the gyroscale fractional cross helicity $\sigma$, the gyroscale Alfvén ratio $r_{\mathrm{A}}$, and the scaling exponent $a$ defined in Equation (15), which characterizes the steepness of the turbulent power spectra at wavenumbers $\sim \rho_{\mathrm{p}}^{-1}$. Our calculation is restricted to values of $\beta_{\| \mathrm{p}}$ that are $\lesssim 1$ (see the discussion preceding Equation (8)).

When applied to alpha particles, our results reproduce three features of the alpha-to-proton perpendicular temperature ratios measured by the Wind spacecraft in weakly collisional solar-wind streams (Kasper et al. 2013), at least for certain values of the model parameters. First, if we set $a=0.25$ (the value that would arise if the electric-field and magnetic-field power spectra were $\propto k_{\perp}^{-3 / 2}$ at $k_{\perp} \rho_{\mathrm{p}} \sim 1$ ), then we find that $6 \lesssim T_{\perp \alpha} / T_{\perp \mathrm{p}} \lesssim 7$ at small $U_{\alpha} / v_{\mathrm{A}}$ when $\beta_{\| \mathrm{p}} \lesssim 1$. Second, when $\beta_{\| \mathrm{p}}$ is small, $T_{\perp \alpha} / T_{\perp \mathrm{p}}$ decreases markedly as $U_{\alpha} / v_{\mathrm{A}}$ increases from 0 to 1 , provided $r_{\mathrm{A}}$ and $\sigma$ are not too small. Third, this decrease becomes less pronounced as $\beta_{\| \mathrm{p}}$ increases to values $\sim 1$. On the other hand, if $|\sigma|$ and/or $r_{\mathrm{A}}$ are reduced sufficiently, then our model produces enhanced values $(\gtrsim 5)$ of $T_{\perp \alpha} / T_{\perp \mathrm{p}}$ at $\left|U_{\alpha}\right| \sim v_{\mathrm{A}}$ that are not seen in the data. The ability of our model to explain the data thus depends on the values of $\sigma$ and $r_{\mathrm{A}}$ in the solar wind. Of all the $T_{\perp \alpha} / T_{\perp \mathrm{p}}$ plots shown in this paper, the one that most closely resembles K13's Figure 2 is our Figure 5 In this panel, $T_{\perp \alpha} / T_{\perp \mathrm{p}}$ is averaged over uniform distributions of $\sigma$ and $r_{\mathrm{A}}$ in which $0.4 \leq|\sigma| \leq 0.8$ and $1.5 \leq r_{\mathrm{A}} \leq 2.5$.

Observationally, the distribution of $\sigma$ and $r_{\mathrm{A}}$ values in the solar wind is not clear. The difficulty is that these quantities depend upon the $\boldsymbol{E}$ and $\boldsymbol{B}$ fluctuations at scales $\sim \rho_{\mathrm{p}}$, and at these scales the $\boldsymbol{E}$ measurements are noisy. That being said, there are some indications that the values of $\sigma$ and $r_{\mathrm{A}}$ in the solar wind are in the range of values in which our model compares well with the data. For example, using Wind data, Podesta \& Bhattachariee (2010) found that the fractional cross helicity is relatively constant within the inertial range, implying that the gyroscale fractional cross helicity $\sigma$ is typically similar to the fractional cross helicity at large lengthscales corresponding, e.g., to $\sim 1$-hour timescales in spacecraft measurements. Fractional cross helicities at $\sim 1$-hour timescales in the range of 0.4 to 0.8 are common in the solar wind at $r=1$ AU (e.g., Roberts et al. 1987; Bavassano et al. 2000; Chen et al. 2013). Regarding the gy- 
roscale Alfvén ratio, measurements from the Cluster spacecraft suggest that $r_{\mathrm{A}}$ is slightly greater than 1 at lengthscales $\sim$ $\rho_{\mathrm{p}}$ and that $r_{\mathrm{A}}$ increases to larger values at even smaller lengthscales (Bale et al. 2005; Salem et al. 2012), a finding that is similar to results from gyrokinetic simulations of lowfrequency plasma turbulence (Howes et al. 2008). However, the results of these studies are not fully conclusive, because of the noise in the electric-field data at scales $\rho_{p}$ and because gyrokinetic simulations have not yet resolved a large enough range of lengthscales bracketing $\rho_{\mathrm{p}}$ to guarantee that the physics at scales $\sim \rho_{p}$ is insensitive to both the largescale driving and grid-scale dissipation. Future observational and/or numerical studies to characterize more precisely the electric-field fluctuations at lengthscales $\sim \rho_{\mathrm{p}}$ would lead to more rigorous tests of the stochastic-heating model.

Further work is also needed to improve our theoretical treatment of stochastic heating. For example, to simplify the calculation, we have assumed that $\sigma$ and $r_{\mathrm{A}}$ are independent of scale at scales $\sim \rho_{\mathrm{p}}$. In the solar wind, however, $r_{\mathrm{A}}$ and possibly $\sigma$ vary with scale. As in Chandran et al. (2010), we have assumed that stochastic heating is dominated by fluctuations at scales comparable to an ion's gyroradius. In reality, however, an ion is likely heated by fluctuations with a range of lengthscales. In addition, we have neglected the possible effects of temperature-anisotropy instabilities. Such instabilities place upper and lower limits on $T_{\perp} / T_{\|}$for both protons and alpha particles, which could affect the value of $T_{\perp \alpha} / T_{\perp \mathrm{p}}$.
These instabilities become increasingly important as $\beta_{\| \mathrm{p}}$ increases and should be included in future models.

Finally, although we have focused on alpha particles and protons, we note that our analysis also applies to minor ions. Observations suggest that minor ions at $r=1$ AU have $w_{\| \mathrm{i}}$ values that are similar to $w_{\| \alpha}$ (Bochsler 2007). Figure 3 therefore approximately describes minor ions as well as alpha particles, provided one adjusts the color scale to account for the change in the normalization of $T_{\perp \mathrm{i}} / T_{\perp \mathrm{p}}$ for different ion species due to the different values of the factor $A(A / Z)^{2 a \eta}$ on the right-hand side of Equation (20). Measurements of $T_{\perp \mathrm{i}} / T_{\perp \mathrm{p}}$ for ions other than alpha particles, and how $T_{\perp \mathrm{i}} / T_{\perp \mathrm{p}}$ depends upon $w_{\| \mathrm{i}}$ and $U_{\mathrm{i}}$, would thus lead to further tests of the stochastic heating model.

We thank A. Schekochihin for helpful discussions and the referee for valuable suggestions. This work was supported by grant NNX11AJ37G from NASA's Heliophysics Theory Program, NASA grant NNN06AA01C to the Solar Probe Plus FIELDS Experiment, NASA grant NNX13AF97G, NSF grant AGS-0851005, NSF/DOE grant AGS-1003451, and DOE grant DE-FG02-07-ER46372. B. Chandran was supported by a Visiting Research Fellowship from Merton College, University of Oxford. E. Quataert was supported by a Simons Investigator award from the Simons Foundation, the David and Lucile Packard Foundation, and the Thomas Alison Schneider Chair in Physics at UC Berkeley.

\section{REFERENCES}

Bale, S. D., Kasper, J. C., Howes, G. G., Quataert, E., Salem, C., \& Sundkvist, D. 2009, Physical Review Letters, 103, 211101

Bale, S. D., Kellogg, P. J., Mozer, F. S., Horbury, T. S., \& Reme, H. 2005 , Physical Review Letters, 94, 215002

Bavassano, B., Pietropaolo, E., \& Bruno, R. 2000, J. Geophys. Res., 105, 15959

Belcher, J. W., \& Davis, Jr., L. 1971, J. Geophys. Res., 76, 3534

Bieber, J. W., Matthaeus, W. H., Smith, C. W., Wanner, W., Kallenrode, M. B., \& Wibberenz, G. 1994, Astrophysical Journal, 420, 294

Bochsler, P. 2007, A\&A Rev., 14, 1

Bourouaine, B., \& Chandran, B. 2013, accepted, Astrophys. J.

Chandran, B. D. G. 2005, Phys. Rev. Lett., 95, 265004

—. 2008b, Phys. Rev. Lett., 101, 235004

-. 2010, ApJ, 720, 548

Chandran, B. D. G., Li, B., Rogers, B. N., Quataert, E., \& Germaschewski, K. 2010, ApJ, 720, 503

Chaston, C. C., Bonnell, J. W., Carlson, C. W., McFadden, J. P., Ergun, R. E., Strangeway, R. J., \& Lund, E. J. 2004, Journal of Geophysical Research (Space Physics), 109, 4205

Chen, C. H. K., Bale, S. D., Salem, C. S., \& Maruca, B. A. 2013, ApJ, 770, 125

Chen, C. H. K., Mallet, A., Schekochihin, A. A., Horbury, T. S., Wicks, R. T., \& Bale, S. D. 2012, ApJ, 758, 120

Chen, L., Lin, Z., \& White, R. 2001, Physics of Plasmas, 8, 4713

Cho, J., \& Lazarian, A. 2002, Phys. Rev. Lett., 88, 245001

Cho, J., \& Lazarian, A. 2004, ApJ, 615, L41

Cranmer, S. R., \& van Ballegooijen, A. A. 2012, ApJ, 754, 92

Dmitruk, P., Matthaeus, W. H., \& Seenu, N. 2004, ApJ, 617, 667

Fiksel, G., Almagri, A. F., Chapman, B. E., Mirnov, V. V., Ren, Y., Sarff, J. S., \& Terry, P. W. 2009, Physical Review Letters, 103, 145002

Galinsky, V. L., \& Shevchenko, V. I. 2000, Physical Review Letters, 85, 90

Gary, S. P., Yin, L., Winske, D., \& Reisenfeld, D. B. 2000, Geophys. Res. Lett., 27, 1355

Goldreich, P., \& Sridhar, S. 1995, ApJ, 438, 763

Hellinger, P., Trávníček, P., Kasper, J. C., \& Lazarus, A. J. 2006, Geophys. Res. Lett., 33, 9101

Hellinger, P., \& Trávníček, P. M. 2011, Journal of Geophysical Research (Space Physics), 116, 11101

Hellinger, P., TráVníčEk, P. M., Å TveráK, Å. T., Matteini, L., \& Velli, M. 2013, Journal of Geophysical Research (Space Physics), 118, 1351
Higdon, J. C. 1984, ApJ, 285, 109

Hollweg, J. V. 1986, J. Geophys. Res., 91, 4111

-. 1999, J. Geophys. Res., 104, 14811

Hollweg, J. V., \& Isenberg, P. A. 2002, Journal of Geophysical Research (Space Physics), 107, 1147

Hollweg, J. V., \& Johnson, W. 1988, J. Geophys. Res., 93, 9547

Horbury, T. S., Forman, M., \& Oughton, S. 2008, Physical Review Letters, 101,175005

Howes, G. G., Bale, S. D., Klein, K. G., Chen, C. H. K., Salem, C. S., \& TenBarge, J. M. 2012, ApJ, 753, L19

Howes, G. G., Dorland, W., Cowley, S. C., Hammett, G. W., Quataert, E., Schekochihin, A. A., \& Tatsuno, T. 2008, Physical Review Letters, 100, 065004

Isenberg, P. A. 1984, J. Geophys. Res., 89, 6613

Isenberg, P. A., \& Hollweg, J. V. 1983, J. Geophys. Res., 88, 3923

Isenberg, P. A., Lee, M. A., \& Hollweg, J. V. 2001, J. Geophys. Res., 106, 5649

Isenberg, P. A., \& Vasquez, B. J. 2007, ApJ, 668, 546

—. 2011, ApJ, 731, 88

Johnson, J. R., \& Cheng, C. Z. 2001, Geophys. Res. Lett., 28, 4421

Karimabadi, H., Omidi, N., \& Gary, S. P. 1994, Washington DC American Geophysical Union Geophysical Monograph Series, 84, 221

Kasper, J. C., Lazarus, A. J., \& Gary, S. P. 2002, Geophys. Res. Lett., 29, 170000

—. 2008, Physical Review Letters, 101, 261103

Kasper, J. C., Maruca, B. A., Stevens, M. L., \& Zaslavsky, A. 2013,

Physical Review Letters, 110, 091102

Kohl, J. L., et al. 1998, ApJ, 501, L127

Kruskal, M. 1962, Journal of Mathematical Physics, 3, 806

Li, X., \& Habbal, S. R. 2001, J. Geophys. Res., 106, 10669

Maron, J., \& Goldreich, P. 2001, ApJ, 554, 1175

Marsch, E., Ao, X.-Z., \& Tu, C.-Y. 2004, Journal of Geophysical Research (Space Physics), 109, 4102

Marsch, E., Rosenbauer, H., Schwenn, R., Muehlhaeuser, K., \& Neubauer, F. M. 1982a, J. Geophys. Res., 87, 35

Marsch, E., Schwenn, R., Rosenbauer, H., Muehlhaeuser, K., Pilipp, W., \& Neubauer, F. M. 1982b, J. Geophys. Res., 87, 52

Matteini, L., Landi, S., Hellinger, P., Pantellini, F., Maksimovic, M., Velli, M., Goldstein, B. E., \& Marsch, E. 2007, Geophys. Res. Lett., 34, 20105 
Matthaeus, W. H., Goldstein, M. L., \& Roberts, D. A. 1990,

J. Geophys. Res., 95, 20673

McChesney, J. M., Stern, R. A., \& Bellan, P. M. 1987, Physical Review Letters, 59, 1436

Parashar, T. N., Shay, M. A., Cassak, P. A., \& Matthaeus, W. H. 2009, Physics of Plasmas, 16, 032310

Podesta, J. J., \& Bhattacharjee, A. 2010, ApJ, 718, 1151

Quataert, E. 1998, ApJ, 500, 978

Roberts, D. A., Goldstein, M. L., Klein, L. W., \& Matthaeus, W. H. 1987, J. Geophys. Res., 92, 12023

Salem, C. S., Howes, G. G., Sundkvist, D., Bale, S. D., Chaston, C. C., Chen, C. H. K., \& Mozer, F. S. 2012, ApJ, 745, L9

Schekochihin, A. A., Cowley, S. C., Dorland, W., Hammett, G. W., Howes, G. G., Quataert, E., \& Tatsuno, T. 2009, ApJS, 182, 310
Servidio, S., Valentini, F., Califano, F., \& Veltri, P. 2012, Physical Review Letters, 108, 045001

Shebalin, J. V., Matthaeus, W., \& Montgomery, D. 1983, Journal of Plasma Physics, 29, 525

Tu, C.-Y., Pu, Z.-Y., \& Wei, F.-S. 1984, J. Geophys. Res., 89, 9695

Verscharen, D., \& Chandran, B. D. G. 2013, ApJ, 764, 88

Wicks, R. T., Horbury, T. S., Chen, C. H. K., \& Schekochihin, A. A. 2010, MNRAS, 407, L31

Xia, Q., Perez, J. C., Chandran, B. D. G., \& Quataert, E. 2013, submitted, Astrophys. J.

Yao, S., He, J.-S., Marsch, E., Tu, C.-Y., Pedersen, A., Rème, H., \& Trotignon, J. G. 2011, ApJ, 728, 146 